\title{
Platypnea-Orthodeoxia Syndrome: Positional Reversal of the In- teratrial Pressure Gradient Demonstrated by Atrial Septal Motion
}

\author{
Serio Farris ${ }^{1}$ and Serio M Kerala ${ }^{2^{*}}$ \\ ${ }^{1}$ Touro University, California, USA \\ ${ }^{2}$ Department of Cardiology, Natividad Medical Center, California, USA
}

*Corresponding author: Serio M Kerala, MD FACC, Department of Cardiology, Natividad Medical Center, 1441 Constitution Blvd, Salinas, CA 93912, California, USA, Tel: +831-796-1630,E-mail: seriom@natividad.com

\begin{abstract}
Platypnea-Orthodeoxia Syndrome (POS) is a rare clinical syndrome characterized by dyspnea and hypoxemia while upright that are relieved when the patient is supine. A right to left shunt in combination with several hypothesized hemodynamic or structural factors are needed to develop the syndrome. It is an important source of dyspnea in a small percentage of often-elderly patients. The exact mechanism for the positional increase in the shunt has remained elusive.

We present the case of an 85-year-old woman with severe POS. The patient has an atrial septal aneurysm with a Patent Foramen Ovale (PFO). We were able to demonstrate a significant increase in the right to left intra-atrial shunt in the upright position using transesophageal echocardiography and agitated saline study. By analyzing the atrial septal motion, we were also able to provide evidence that the shunt is caused by a reversal in the inter-atrial pressure gradient in the upright position, thus we are able to show for the first time that a reversal of the interatrial pressure gradient can be responsible for the positional shunt.
\end{abstract}

\section{Keywords}

Platypnea-orthodeoxia syndrome, Patent foramen ovale, Positional desaturation

\section{Case Report}

An 85-year-old woman who presented with severe progressive shortness of breath over 3 months. These symptoms had become so severe that she had to dress herself and have her meals while supine in bed. Her medical history was significant for single-vessel coronary artery disease with a previous stent placement, hypertension, hyperlipidemia, anxiety, and hypothyroidism.
CT angiogram was negative for pulmonary emboli. Because of her persistent hypoxemia she was transferred to our medical center where her oxygen saturation was noted to be $98 \%$ while supine and $75 \%$ while upright. A Transthoracic Echocardiogram (TTE) bubble study was performed and showed normal left ventricular size and contractility with an ejection fraction of $65 \%$. Also noted were mild aortic insufficiency, tricuspid regurgitation, and normal pulmonary artery systolic pressure. There was a small right-to-left shunt. A CT angiogram showed no pulmonary emboli, pulmonary arteriovenous shunting, or dissection. A Transesophageal Echocardiogram (TEE) was performed. No intra-atrial shunt was seen by color Doppler in left lateral position, the agitated saline study showed minimal right to left atrial shunting, and no defect was visualized. Next, the head of the bed was raised to $70^{\circ}$ with the patient in the same left lateral position, and another agitated saline study was performed. This study demonstrated a severe right to left shunt through a patent foramen ovale at the lower margin of the fossa ovale with separation of the septa by 7 $\mathrm{mm}$ (Figure 1). Within 2-3 minutes of raising the head of the bed, the patient's oxygen saturation dropped from $98 \%$ to $85 \%$ on high flow oxygen.

Based on the clinical and echocardiographic findings, it was determined that the patient had significant cardiac POS and required PFO closure.

She was transferred to another facility that has a cardiac catheterization laboratory. There she underwent right and left cardiac catheterization, which demon-

Citation: Farris S, Kerala SM (2017) Platypnea-Orthodeoxia Syndrome: Positional Reversal of the Interatrial Pressure Gradient Demonstrated by Atrial Septal Motion. Int J Clin Cardiol 5:107. doi. org/10.23937/2378-2951/1410107

Received: October 27, 2017; Accepted: January 09, 2018; Published: January 11, 2018

Copyright: (C) 2018 Farris S, et al. This is an open-access article distributed under the terms of the Creative Commons Attribution License, which permits unrestricted use, distribution, and reproduction in any medium, provided the original author and source are credited. 


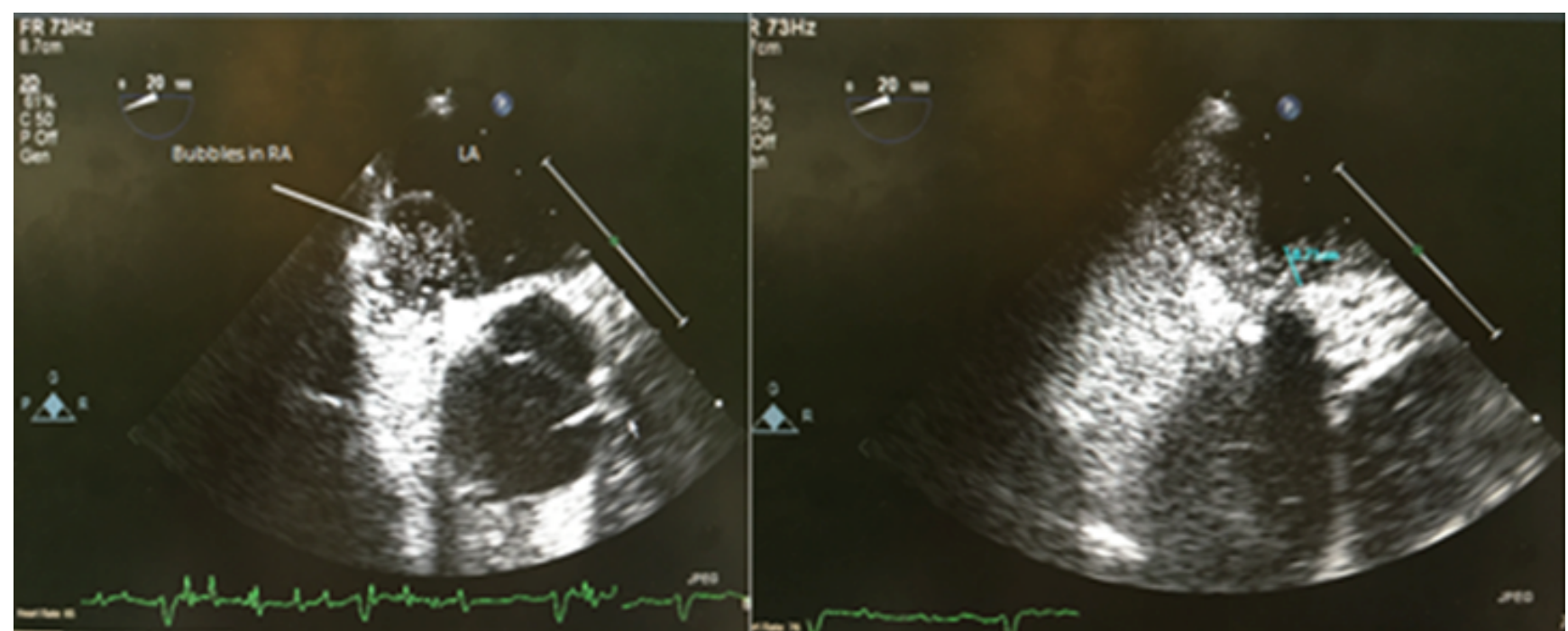

Figure 1: Agitated saline study showing minimal shunt in the supine position (Left) and a severe right to left atrial shunt with a $7 \mathrm{~mm}$ shunt width when the patient was elevated to 70 degrees (Right).

strated that her prior stent to be patent, no evidence of other obstructive coronary artery disease, and normal right side cardiac pressures.

She underwent surgical repair of the septal defect at the same facility. Immediately after extubation, it was noted that her orthostatic desaturation had resolved. The patient was discharged shortly thereafter to a rehabilitation program. At one month follow up, she remained asymptomatic.

\section{Findings}

Our patient was found to have a redundant atrial septum consistent with an Atrial Septal Aneurysm (ASA). The redundancy of the atrial septum allowed for frame by frame analysis of the septal motion throughout the cardiac cycle. This was done both in the supine and upright positions (Figure 2).

In the supine position, the atrial septum had a bidirectional motion with maximum excursion towards the left atrium. In the upright position, the septal motion changed and its deflection was maintained towards the left atrium throughout the cardiac cycle.

\section{Discussion}

Platypnea-Orthodeoxia Syndrome is characterized by arterial desaturation that persists while erect, and is relieved by recumbency. For the syndrome to occur, two components must be present. The first component is an arteriovenous shunt, and the second component is a proposed mechanical or hemodynamic factor that causes the shunt to occur or worsen in the upright position.

The contribution of the intracardiac shunt to the cardiac POS is straightforward. Its presence is mandatory for the development of the syndrome, it is usually easy to demonstrate by echocardiography and by fixing it, and the patient's desaturation and symptoms improve immediately.

On the other hand, the second component for cardiac POS to occur in the upright position has been the main challenge in understanding and diagnosing the syndrome. Multiple mechanisms have been proposed; all were theoretical, and most could not be proven. These factors were almost always categorized into two main groups, a mechanical group and a hemodynamic group.

\section{The mechanical group}

Multiple anatomical factors were implicated, including but not limited to aortic dilation, kyphoscoliosis, atrial septal aneurism and a prominent Eustachian valve. These factors were theorized to cause an increased shunt in the upright position by distorting the atrial septal morphology, changing its diameter, or redirecting venous blood flow towards septum [1]. The main challenge in showing a structural or anatomical difference between the upright and supine position by CT or MRI imaging is the limitation of the patient to the horizontal position. Also most cases reported symptoms evolving over a short period of time, which is inconsistent with the chronicity of the proposed etiologies.

\section{The hemodynamic group}

The hemodynamic group suggested a transient change in the interatrial gradient leading to the shunt in the upright position. Different comorbidities such as pericarditis, COPD, hypotension or pneumonectomy have been described. Yet normal variation of chamber pressure based on positioning have not been assessed or reported in the literature.

Based on the study performed by Kusumoto, et al. [2] the overall inter-atrial septal shape depends on the pressure gradient between the left and right atria. They showed that age, arterial blood pressure and heartrate 


\section{Supine}

\section{Upright}

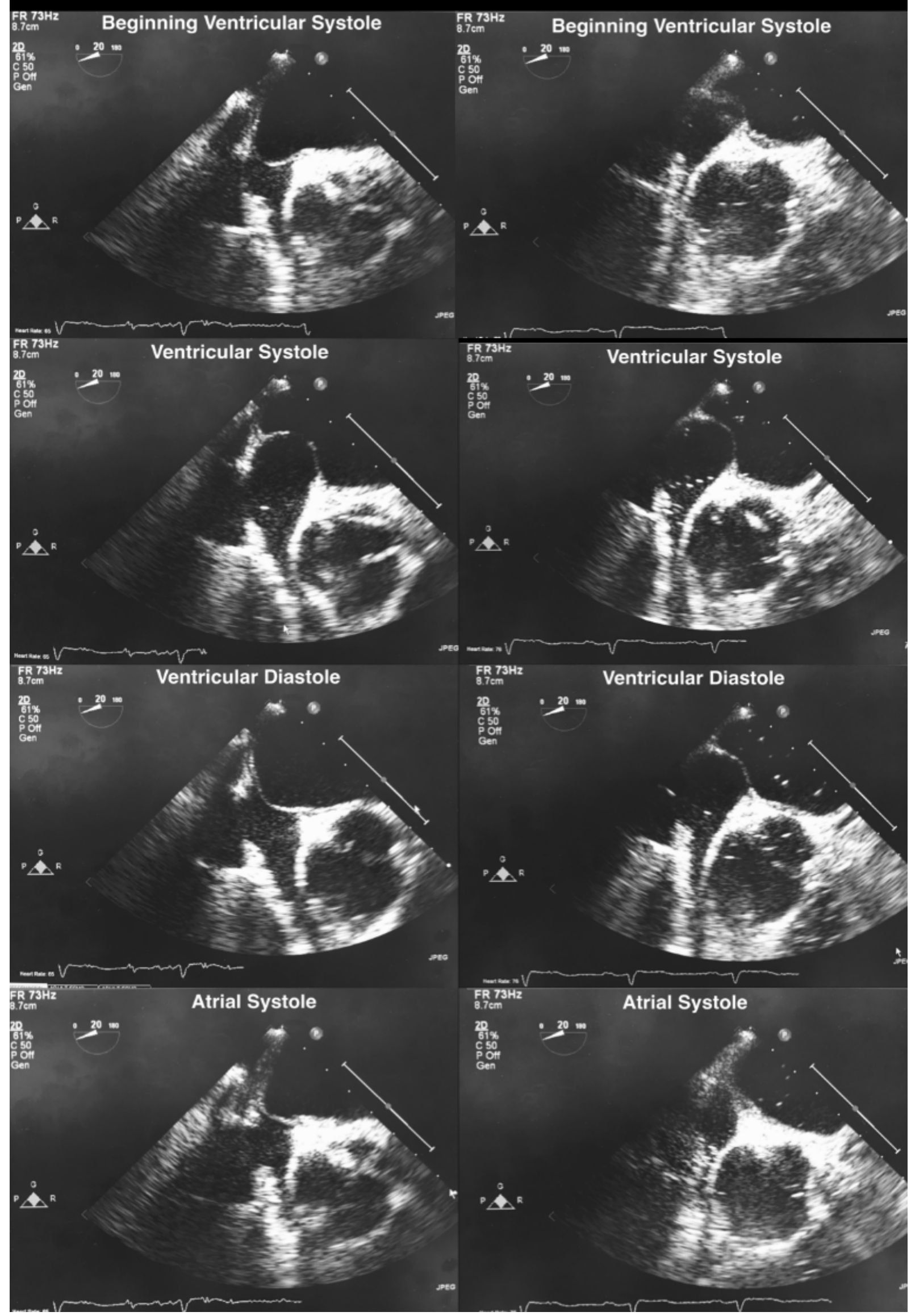

Figure 2: Comparison of the atrial septal motion between supine and upright positions at four different points in the cardiac cycle. Top row: Beginning of ventricular systole. Second row: Ventricular systole. Third row: Ventricular diastole. Bottom row: Atrial systole. The rhythm strip on the bottom of each panel identifies the timing of acquisition during the cardiac cycle. 
had no discernible effect on the curvature or excursion of the interatrial septum. Yonezawa, et al. [3] had also previously shown that the motion of the atrial septum is considered to be dependent on the inter-atrial pressure gradient except during atrial contraction.

Based on these studies, the atrial septal motion in our patient in the supine position was consistent with normal interatrial pressure gradient. In the upright position, the displacement of the interatrial septum to the left was consistent with a reversal in the interatrial pressure gradient. This explains the sudden and significant increase of right-to-left intracardiac shunt with immediate desaturation noted in our patient in the upright position. To our knowledge and based on our review of the literature, the use of the atrial septal motion to show a change in the interatrial pressure gradient leading to an increased shunt in the upright position, has not been described before.

The mechanism for the change in the interatrial atrial pressure gradient in the upright position is still not clear. In a simulated obstructive sleep apnea study, Konecny, et al. [4] showed that right atrial pressure transiently and consistently exceeded left atrial pressure in response to the steep decline in intrathoracic pressure during a Mueller maneuver. There have been no studies to show the effect of upright position on intrathoracic pressure, thus a potential relationship with POS.
Patients who undergo neurosurgical procedures performed in the sitting position, have a higher risk of paradoxical venous emboli through PFO's [5] which might be also related to a change in the interatrial gradient in the upright position. The patients who had significant paradoxical air emboli in the study had higher pulmonary artery pressure during these episodes.

It had been suggested that a mechanical distortion of the atrial septum occurring in the upright position leads to the increased shunt [6]. In our patient we measured the diameter of the fossa ovalis both in the supine and upright positions, at the level of the coronary sinuses with 20 degree sector rotation. We compared the maximum and minimum fossa ovalis diameters as well as their ratios in both positions (Figure 3). In the supine position the maximum diameter was $2.19 \mathrm{~cm}$ and the minimum was $1.08 \mathrm{~cm}$ with a ratio of 0.49 . In the upright position the maximum diameter was $2.12 \mathrm{~cm}$ and the minimum was $1.14 \mathrm{~cm}$ with a ratio of 0.53 . Thus, at least in our patient, we could not show a size difference of the fossa ovale between the supine and upright positions that might lead to redundancy of the atrial septum and an increase in the shunt.

Bertaux, et al. [6] found a correlation between aortic root diameter and atrial septal dimension and oscillation amplitude. They also found that patients with grade 3 intracardiac shunting through a PFO possibly had lower interatrial pressure gradient values than oth-

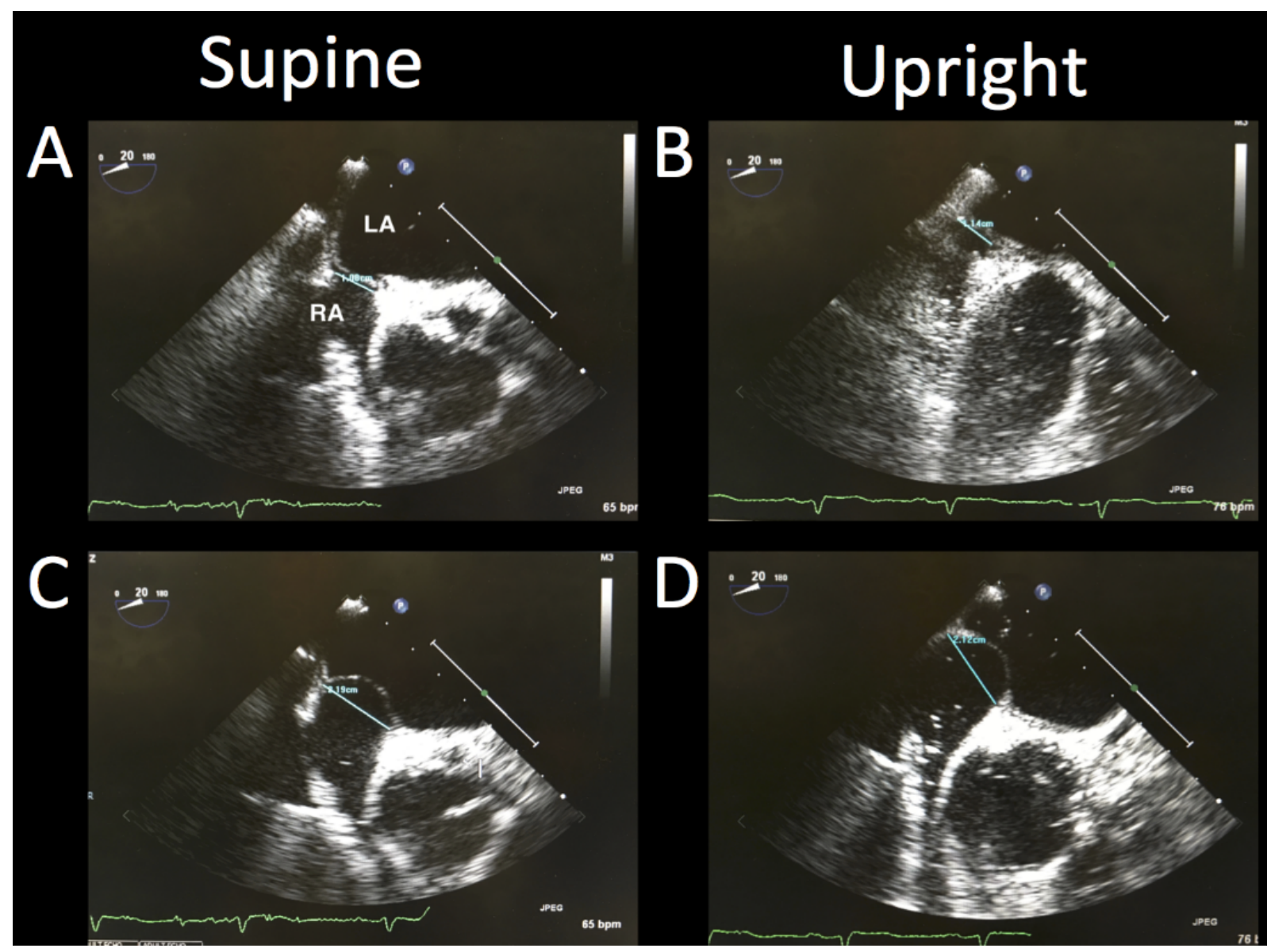

Figure 3: Minimum: $A, B$ and Maximum: $C, D$ fossa ovalis diameter in the supine and upright positions. 


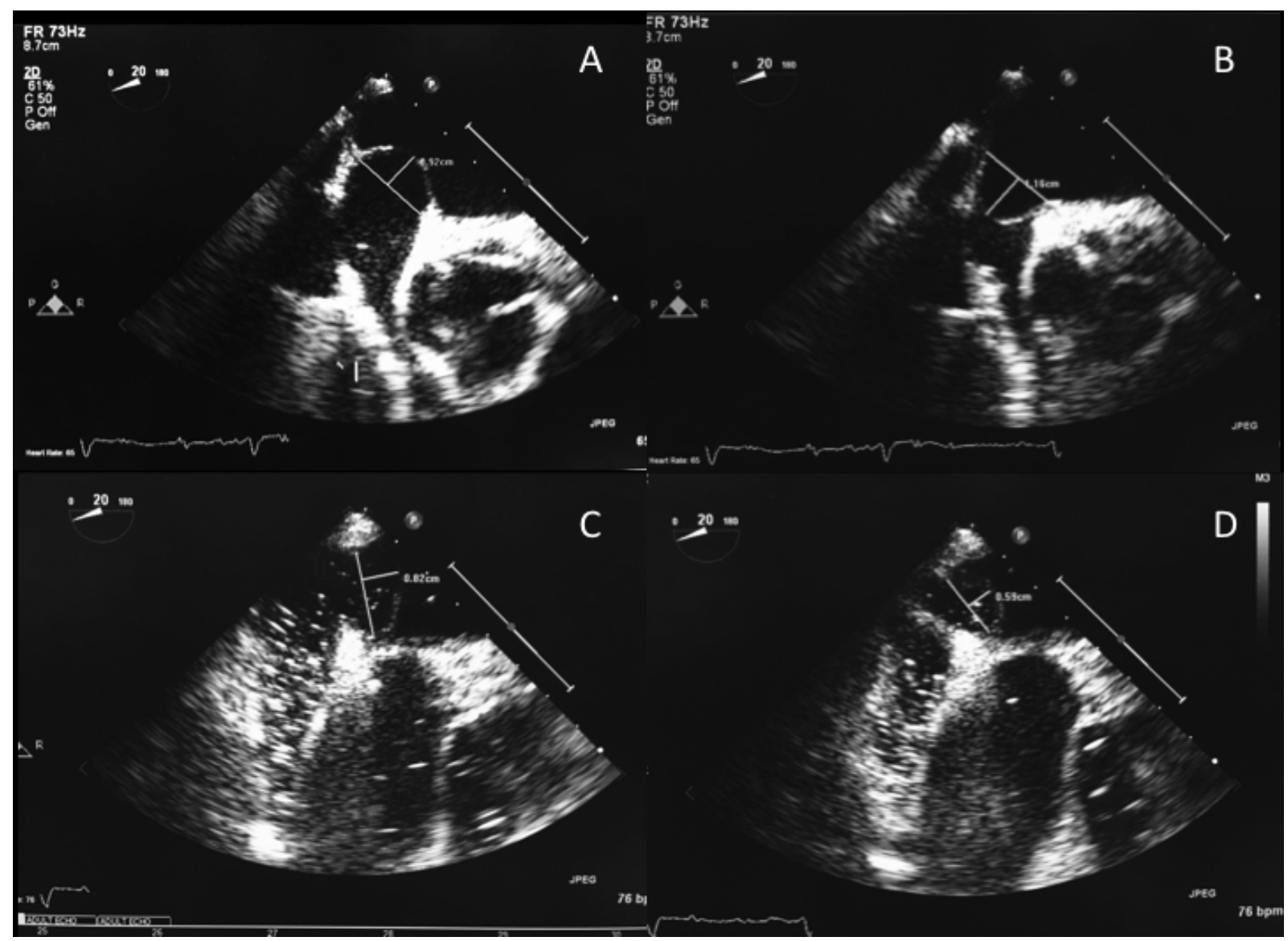

Figure 4: Atrial septal oscillation in supine and upright positions A) Maximum left deflection supine; B) Maximum right deflection supine; C) Maximum left deflection upright; D) Maximum right deflection upright.

er patients in their study. The study did not assess the effect of the upright position on any of these parameters, thus it is not possible to make conclusions in relation to POS patients. In our patient the maximum atrial septal oscillation actually decreased in the upright compared to the supine position, at the same time when the shunt had significantly increased and without a change in the fossa ovalis diameter (Figure 4). This suggests that the amplitude of the atrial septal oscillation is not necessarily related to the diameter of the fossa ovalis or the degree of shunt. The shunt seems to be, at least in this case, related to a reversal of the interatrial pressure gradient. We argue that without this change in the interatrial pressure gradient, the atrial septal oscillation should have kept the same pattern in both upright and supine positions, regardless of the degree of oscillation.

\section{Limitations}

The limitations in our study are obvious. This is a single case report. The hemodynamic obtained during cardiac catheterization was performed only in the supine position. Also, there have been no studies in healthy patients of positional changes in interatrial pressure gradient to compare with the findings in our patient.

In the future, healthy patients who have PFO's can be further studied using transesophageal echocardiography in the supine and upright position, to assess the normal effect of body position on the degree of shunt and examine the atrial septal motion and interatrial atrial pressure gradient.

Patients with POS who undergo transesophageal echocardiograms can have the atrial septal motion and the inter-atrial pressure gradient analyzed in the supine and upright positions to further examine our finding.

Patients with documented POS can be studied with invasive cardiac hemodynamics in the supine and upright positions to shed more light on the mechanism of this syndrome.

Intrathoracic pressure measurements in the supine and upright positions and their relationship to interatrial pressure gradient can be studied, both to assess the effect of position on intrathoracic pressure, and to assess for a potential link between the intrathoracic pressure and the increased interatrial gradient in the upright position.

\section{Conclusion}

Cardiac platypnea-orthodeoxia is a rarely diagnosed but clinically important syndrome. It requires an intracardiac shunt, usually through a PFO, which increases in the upright position, leading to shortness of breath and desaturation. The difficulties so far have been in recognizing the mechanism that leads to the postural increase in the shunt. Multiple mechanical and hemodynamic factors have been implicated. A change in the in- 
teratrial pressure gradient in the upright position might cause the increase in the right to left intracardiac shunt in some patients. By analyzing the atrial septal motion in the supine and upright positions in our patient with severe POS, we were able to show for the first time that the increase in the right to left intracardiac shunt in the upright position is directly related to, and most likely caused by a reversal of the interatrial pressure gradient.

Further studies are needed to establish the mechanism by which the interatrial pressure gradient changes in some patients leading to cardiac POS.

\section{References}

1. Akin E, Kruger U, Braun P, Stroh E, Janicke I, et al. (2014) "The Platypnea-orthodeoxia Syndrome". European Review for Medical and Pharmacological Sciences 18: 2599-2604.

2. Kusumoto FM, Muhiudeen IA, Kuecherer HF, Cahalan MK, Schiller NB (1993) Response of the interatrial septum to transatrial pressure gradients and its potential for predicting pulmonary capillary wedge pressure: An intraoperative study using transesophageal echocardiography in patients during mechanical ventilation. J Am Coll Cardiol 21: 721728 .

3. Yonezawa F, Matsuzaki M, Anno Y, Toma Y, Hiroyama $\mathrm{N}$, et al. (1987) Relationship between interatrial pressure gradient and motion of the interatrial septum. J Cardiol 17: 617-623.

4. Konecny T, Khanna AD, Novak J, Abdi A Jama, George M Zawadowski, et al. (2014) Interatrial pressure gradients during simulated obstructive sleep apnea: A catheter-based study. Catheterization and Cardiovascular Interventions 84: 1138-1145.

5. Mammoto T, Hayashi Y, Ohnishi Y, Kuro M (1998) Incidence of venous and paradoxical air embolism in neurosurgical patients in the sitting position: Detection by transesophageal echocardiography. Acta Anaesthesiologica Scandinavica 42: 643-647.

6. Bertaux G, Eicher JC, Petit A, Dobšák P, Wolf JE (2007) Anotomic Interaction Between the Aortic Root and the Atrial Septum: A Prospective Echocardiographic Study. J Am Soc Echocardiogr 20: 409-414. 